\title{
Erratum: Heat Flow Between Nuclei and Conduction Electrons in Metals at Very Low Temperature*
}

\author{
Georg Eska
}

Physikalisches Institut, Universität Bayreuth, D-8580 Bayreuth, Germany

There is an error in (8) that should be mentioned. Equations (8a) and (8b) should read as follows:

$$
\hat{\beta}_{n}=\left(\beta_{e}-\beta_{n}\right) / \tau_{n e}-\beta_{n} \beta_{1} / B_{1}
$$

and

$$
\hat{\beta}_{N}=\left(\beta_{E}-\beta_{N}\right) / \tau_{N E}-\beta_{N} \stackrel{\circ}{B}_{2} / B_{2}
$$

*This paper appeared in J. Low Temp. Phys. 88, 273 (1992). 